\title{
L'hépatite C au Canada et l'importance du dépistage fondé sur les risques
}

\author{
Ha $\mathrm{S}^{1 *}$, Totten $\mathrm{S}^{1}$, Pogany $\mathrm{L}^{1}$, Wu J1', Gale-Rowe $\mathrm{M}^{1}$
}

\section{Résumé}

L'hépatite $\mathrm{C}$ chronique demeure un problème de santé publique qui touche environ 220000 personnes au Canada. En 2011, environ $44 \%$ des personnes souffrant d'hépatite C chronique ne savaient pas qu'elles étaient infectées. L'hépatite $C$ est d'origine infectieuse et, si elle n'est pas traitée, peut entraîner une morbidité et une mortalité importantes sous sa forme chronique, notamment une cirrhose, un carcinome hépatocellulaire et une insuffisance hépatique. Ces résultats en matière de santé sont associés à des comorbidités, ce qui ajoute un poids supplémentaire au système de santé canadien. De récentes avancées dans le traitement de l'hépatite $C$ ont modifié le paysage clinique.

Au Canada, la prévalence des nouveaux cas est plus élevée chez certains groupes de populations. L'usage de drogues injectables représente actuellement la majorité des nouvelles infections par le virus de l'hépatite $\mathrm{C}(\mathrm{VHC})$. Nous ne savons pas précisément dans quelle mesure l'infection par le VHC associée à l'utilisation de services de soins de santé ou personnels a contribué aux cas prévalents d'hépatite $C$ chronique. Le Groupe d'étude canadien sur les soins de santé préventifs (GECSSP) examine actuellement les données probantes relatives à différentes approches de dépistage du VHC, ainsi que les avantages et inconvénients d'un tel dépistage. Le dépistage fondé sur les risques demeure essentiel pour détecter l'hépatite $C_{\text {, }}$ car la connaissance du statut d'une personne a été associée au parcours de soins et à une amélioration des résultats de cette population en matière de santé. Le présent article vise à mettre en évidence les facteurs de risque associés à l'acquisition du VHC afin que les fournisseurs de soins de santé puissent dépister, s'il y a lieu, et détecter les cas d'hépatite C chronique.

\begin{abstract}
Affiliations
${ }^{1}$ Centre de la lutte contre les maladies transmissibles et les infections, Agence de la santé publique du Canada, Ottawa (Ontario)
\end{abstract}

*Correspondance : shalane.ha@ phac-aspc.gc.ca

Citation proposée: Ha S, Totten S, Pogany L, Wu J, Gale-Rowe M. L'hépatite C au Canada et l'importance du dépistage fondé sur les risques. Relevé des maladies transmissibles au Canada 2016;42:65-71.

https://doi.org/10.14745/ccdr.v42i03a02f

\section{Introduction}

L'hépatite $C$, une inflammation du foie causée par le virus de I'hépatite $\mathrm{C}(\mathrm{VHC})$, est d'origine infectieuse et peut provoquer une maladie chronique à laquelle s'ajoute la dimension de transmission. L'hépatite $C$ est souvent asymptomatique pendant des décennies, jusqu'à ce que les dommages causés au foie se fassent sentir; d'où l'importance d'un dépistage précoce.

Le contact par le sang avec une personne ou un produit infectés constitue son principal mode de transmission. Le VHC peut provoquer une hépatite $C$ aiguë et chronique. Environ $25 \%$ des personnes souffrant d'hépatite $C$ aiguë élimineront spontanément le virus (1); les $75 \%$ restant contractent une hépatite $\mathrm{C}$ chronique qui peut se transformer en cirrhose, en insuffisance hépatique ou en carcinome hépatocellulaire (2).

Parmi les sept génotypes de VHC connus dans le monde (3), le génotype 1 est le plus courant au Canada (4) et a été difficile à traiter jusqu'à récemment. Les approbations de nouveaux médicaments pour la prise en charge de l'hépatite $C$ chronique au Canada peuvent faire de celle-ci une maladie infectieuse guérissable.

Au Canada, 346 décès ont été attribués à I'hépatite $C$ chronique en 2011 (5); toutefois, le nombre de décès liés à l'hépatite $C$ est probablement sous-estimé de façon considérable en raison de la classification erronée sur les certificats de décès (6). Les données du Registre canadien des insuffisances et des transplantations d'organes (RCITO) indiquent que le virus de I'hépatite C était le principal diagnostic de $21 \%$ des personnes ayant bénéficié d'une transplantation du foie, de 2004 à 2013, au Canada (7). Dans une étude menée en Ontario en 2010, il a été estimé que le VHC représentait le plus important fardeau des pathogènes infectieux en ce qui concerne le nombre d'années de vie perdues en raison d'une mortalité précoce, les années équivalentes de fonctionnement réduit et les années de vie ajustées en fonction de la santé (8), ce qui souligne l'impact de cette infection sur la santé des Canadiens.

En 2012 et en 2013, les Centers for Disease Control and Prevention (CDC) et le United States Preventive Services Task Force (USPSTF) ont publié des lignes directrices à jour sur le dépistage du VHC. Ils ont recommandé à tous les adultes nés 
entre 1945 et 1965 (génération du baby-boom) de passer un test de dépistage ponctuel du VHC, peu importe leurs facteurs de risque $(9,10)$. Les recommandations des États-Unis visant le dépistage ponctuel des personnes de la génération du baby-boom étaient fondées sur des données épidémiologiques démontrant 1) une forte prévalence du VHC dans la cohorte de naissance de la génération du baby-boom, 2) une proportion élevée de patients atteints d'une infection non diagnostiquée, 3) une augmentation prévue du fardeau de la maladie et 4) des données probantes indirectes liant le dépistage à l'amélioration des résultats en matière de santé $(9,10)$.

À la suite de la publication des recommandations des États-Unis, l'Agence de la santé publique du Canada a examiné le fardeau prévu lié à l'hépatite $C(11)$ et a modélisé les estimations en matière de prévalence de l'anti-virus de I'hépatite $C$ et de l'hépatite $C$ chronique (12). Compte tenu de I'incertitude entourant les estimations et des avantages et des conséquences du dépistage, d'autres analyses étaient requises pour établir si des modifications devraient être apportées aux recommandations actuelles du Canada en matière de dépistage reposant sur le risque. Ces éléments seront examinés par le Groupe d'étude canadien sur les soins de santé préventifs (GECSSP). Le GECSSP effectue un examen des données canadiennes afin de déterminer l'applicabilité des différentes approches de dépistage, notamment le dépistage des cohortes de naissance, au Canada.

Les objectifs du présent article consistent à mettre en évidence les facteurs de risque qui contribuent à la transmission et à I'acquisition du VHC et à encourager les fournisseurs de soins de santé à évaluer le besoin de dépistage du VHC dans le cadre de soins médicaux de routine tandis que nous attendons les résultats de l'évaluation des données probantes pour le dépistage des cohortes de naissance au Canada.

\section{Prise en charge des médicaments}

L'objectif du traitement de I'hépatite $C$ consiste à obtenir une réponse virologique soutenue, définie par l'obtention d'une charge virale non détectable dans le sang à la suite du traitement. Jusqu'à tout récemment, le traitement de l'hépatite $C$ consistait en des injections d'interféron pégylé- $\alpha$ et de ribavirine, ce qui provoquait une réponse virologique soutenue inférieure à 60 \% (13). En outre, les thérapies duraient longtemps (jusqu'à 48 semaines), consistaient en des injections et avaient des effets indésirables importants ( $p$. ex. anémie, fièvre), ce qui limitait l'efficacité et la tolérabilité chez les patients $(13,14)$.

En décembre 2014, Santé Canada a approuvé de nouveaux traitements de courte durée sans interféron (15). Les patients de génotype 1 traités avec ces nouveaux schémas peuvent atteindre une réponse virologique soutenue supérieure à $90 \%$ avec peu d'effets secondaires $(14,16,17)$. Ces nouveaux traitements permettent de réduire la morbidité et la mortalité associées à l'hépatite $C$ et le poids sur le système de soins de santé. L'introduction de nouveaux traitements a permis de passer d'une mentalité où les patients devaient bien vivre avec l'hépatite $C$ à celle que l’hépatite $C$ pouvait être guérie.

\section{Épidémiologie}

Au Canada, le VHC est à déclaration obligatoire sous surveillance nationale depuis 1991. Les autorités sanitaires provinciales et territoriales déclarent des cas d'infection par le VHC au Système canadien de surveillance des maladies à déclaration obligatoire. Les dernières données de surveillance indiquent qu'entre 2005 et 2012 , le taux de cas déclarés de VHC a constamment diminué; il est passé de 40,4 à 29,3 pour 100000 habitants. Les taux ont diminué chez les hommes et les femmes de tous les groupes d'âge, à l'exception de faibles augmentations chez les hommes âgés de 60 ans et plus et les femmes de 25 à 29 ans. Au cours de la période de huit ans, les taux de cas déclarés de VHC ont été systématiquement plus élevés chez les hommes que chez les femmes dans l'ensemble (18). Même si aucun renseignement sur le statut de l'hépatite $C$ aiguë ou chronique n'était disponible auprès des provinces et territoires, cette infection représente probablement la majorité des cas déclarés au Système canadien de surveillance des maladies à déclaration obligatoire, car l'infection aiguë est généralement asymptomatique et il est moins probable d'établir un diagnostic pour celle-ci (18).

Une analyse des effets des cohortes parmi les cas de VHC déclarés entre 1991 et 2010 au Canada a révélé que les personnes nées entre 1946 et 1965 représentaient plus de la moitié de tous les cas (19). Même si le taux de cas déclarés au Canada semble être en baisse, le nombre de personnes infectées il y a quelques décennies et qui vivent maintenant avec des séquelles devrait augmenter au fil du temps à mesure que la maladie progresse (20).

Les études de prévalence de l'infection par le VHC basées sur la population fournissent des renseignements supplémentaires sur l'étendue de la maladie. L'Enquête canadienne sur les mesures de la santé (ECMS) a révélé que la séroprévalence des anticorps contre le $\mathrm{VHC}$ (anti-VHC), un paramètre de l'exposition à vie au virus, était de 0,5\% parmi la population vivant dans un ménage au Canada durant une période de collecte de données s'étalant de 2007 à 2011 (21). Toutefois, les estimations modélisées de la prévalence tenant compte des populations vulnérables non interrogées par l'ECMS (comme les sans-abri, les détenus et les personnes nées à l'étranger qui ne parlent pas l'anglais ou le français) indiquent que le taux de l'anti-VHC au sein de la population canadienne pourrait être plus près de $1 \%$ (intervalle de plausibilité de $0,6 \%$ à 1,3\%) (12). On estime que la prévalence de l'hépatite $C$ chronique est de $0,6 \%$ et qu'environ $44 \%$ des personnes ne savent pas qu'elles sont infectées (12).

\section{Facteurs de risque}

Les renseignements sur les facteurs de risque disponibles grâce à la surveillance nationale du VHC sont limités; toutefois, il existe un nombre considérable de recherches sur les conditions qui influencent la probabilité de devenir infecté par le virus. Vous trouverez ci-dessous un aperçu des pratiques qui augmentent le risque de contracter le $\mathrm{VHC}$, ainsi que les populations déclarées comme ayant des taux d'infection par le VHC plus élevés que la moyenne. 


\section{Utilisation de drogues injectables}

L'utilisation de drogues injectables (UDI) continue d'être la principale cause de cas incidents de VHC dans les pays développés (19). Au Canada, parmi les nouveaux cas d'infection par le virus de l'hépatite $C$ possédant des renseignements en matière de facteurs de risque connus, $61 \%$ avaient des antécédents d'utilisation de drogues injectables (22). De plus, une enquête réalisée entre 2010 et 2012 auprès de personnes utilisant des drogues injectables révélait que $68 \%$ d'entre elles avaient des preuves d'exposition au VHC durant leur vie (23). La prévention de la transmission du VHC est un véritable défi en raison de la forte prévalence de l'infection par le VHC chez les utilisateurs de drogues injectables et de la forte infectiosité du VHC $(24,25)$. L'utilisation de drogues injectables ou le partage d'aiguilles ou d'autre matériel de consommation de drogue (filtres, seringues, pipes, cuillères), ne serait-ce qu'une seule fois, augmente le risque de contracter ou de transmettre I'hépatite C.

\section{Matériel contaminé utilisé dans le cadre de services médicaux, dentaires ou personnels}

Le manque de mise en œuvre de pratiques normalisées de contrôle et de prévention des infections dans le cadre des soins de santé (p. ex. dans les hôpitaux, les cabinets dentaires) ou de services personnels ( $p$. ex. salons de tatouages et de manucure) est un autre risque d'infection par le virus de l'hépatite C (26-30). Par exemple, les patients qui nécessitent une hémodialyse courent un risque plus élevé de contracter le VHC, surtout si le matériel a été réutilisé ou mal stérilisé (26). De plus, un examen systématique récent des publications internationales a révélé que les travailleurs de la santé en contact direct avec des patients ou du sang (blessures par piqûre d'aiguille) ont un risque 1,6 fois plus élevé d'être infectés par le VHC que la population générale. On a trouvé une prévalence accrue du VHC chez les membres du personnel médical (rapport de cotes de 2,2), du personnel de laboratoire (rapport de cotes de 2,2) et du personnel dentaire (rapport de cotes de 3,5) par rapport au groupe témoin (27). Des éclosions de VHC ont également eu lieu dans des cliniques de colonoscopie au Canada (31-35).

Les pratiques de tatouage non sécuritaires utilisant un équipement non stérile augmentent le risque de contracter le VHC (28). Les résultats d'un examen systématique indiquent que le risque de $\mathrm{VHC}$ augmente à mesure que la surface recouverte de tatouages et le nombre de tatouages augmentent (28).

Pour réduire le risque de transmission de maladies infectieuses dans les établissements de soins de santé et de prestation de services personnels, il faut assurer une bonne sensibilisation et une bonne formation du personnel afin qu'il respecte les pratiques de prévention et de contrôle des infections $(29,30)$.

\section{Sang, produits sanguins et greffe d'organe avant 1992}

Avant la mise en œuvre du dépistage systématique dans les dons de sang, les produits sanguins et les organes en 1992, les procédures de transfusion sanguine et de greffe d'organe représentaient un risque important de transmission du virus de I'hépatite $C$. Ces risques ont été minimisés au Canada grâce au dépistage systématique et à l'élimination de dons contenant une maladie transmissible; toutefois, ce n'est pas le cas pour tous les pays. Les données de la Société canadienne du sang de 2014 indiquent que le VHC a été détecté dans 6,1 dons sur 100 000; le risque résiduel de contracter le VHC par réception d'une unité de sang est considéré comme étant très faible et a lieu une fois sur 6,7 millions de dons (36).

\section{Transmission verticale}

La transmission verticale ou d'une mère à son enfant du VHC peut avoir lieu lorsqu'un enfant naît d'une mère infectée par le VHC. Les résultats d'une récente méta-analyse laissent entendre que le risque de transmission verticale d'une femme séropositive pour l'anti-virus de l'hépatite $C$ et séropositive pour l'ARN est d'environ $5,8 \%$ (IC à $95 \%, 4,2 \%$ à $7,8 \%$ ) et que le risque pour les enfants nés d'une mère séropositive pour l'anti-virus de l'hépatite $C$ avec une résolution spontanée de l'infection (négative pour l'ARN) n'étaient pas significatifs $(37,38)$.

\section{Autres activités pouvant entraîner un contact avec du sang contaminé}

Le partage d'articles de soins personnels tels que des rasoirs, des brosses à dents ou des coupe-ongles avec une personne séropositive pour le $\mathrm{VHC}$ représente un risque, même s'il est moins courant, de contracter l'hépatite $C$, car le VHC peut demeurer infectieux sur les surfaces inanimées pendant 6 semaines (24).

Bien que le risque de transmission sexuelle du VHC pendant les relations sexuelles soit faible (39), adopter un comportement sexuel risqué, tel que les relations sexuelles anales sans condom, pourrait transmettre le VHC (40-42) si la muqueuse se rompt, entraînant un contact de sang à sang.

\section{Populations affichant une prévalence plus élevée d'infection par le virus de l'hépatite C}

Hommes ayant des relations sexuelles avec d'autres hommes

Certaines études ont révélé des taux plus élevés d'infection par le VHC chez les hommes ayant des relations sexuelles avec d'autres hommes (HARSAH) séropositifs pour le VIH par rapport aux HARSAH séronégatifs pour le VIH $(43,44)$. Certains HARSAH séropositifs pour le VIH sont plus susceptibles d'adopter des pratiques augmentant le risque de transmission du VHC. Ces pratiques peuvent inclure un comportement sexuel à haut risque (p. ex. relation sexuelle anale non protégée ou pénétration avec le poing, pouvant provoquer un saignement); l'utilisation de drogues injectables et la consommation de drogues à usage récréatif ayant été associées à une activité sexuelle à risque élevé et le sérotriage (c'est-à-dire le fait de choisir des partenaires sexuels ayant la même sérologie $\mathrm{VIH}$ que la sienne) ayant aussi été associé à la transmission d'infections transmissibles sexuellement et par le sang chez les HARSAH séropositifs pour le VIH. En outre, I'infection au VIH elle-même, ainsi que la co-infection avec $d^{\prime}$ 'autres infections transmissibles sexuellement (p. ex. la syphilis, la gonorrhée ou la chlamydiose), peuvent augmenter la probabilité de contracter le VHC par ulcération des muqueuses génitales ou suppression du système immunitaire $(41-43,45-47)$. 


\section{Populations incarcérées}

On a observé des taux élevés d'infection par le virus de l'hépatite $C$ chez les détenus des pénitenciers fédéraux et provinciaux grâce à des dépistages systématiques offerts. Même si tous les détenus n'acceptent pas le dépistage du VHC, ceux ayant reçu un diagnostic d'hépatite $C$ chronique sont traités en fonction de leur stade de fibrose du foie (48). De récentes données de surveillance du Service correctionnel du Canada indiquent que $86,1 \%$ des détenus des pénitenciers fédéraux acceptaient le dépistage du VHC au moment de leur admission et que 18,5 \% étaient séropositifs pour le VHC en 2012 (49). Les facteurs de risque pour la contraction du VHC chez les détenus incluent le contact avec du sang infecté lors de bagarres, l'exposition sexuelle, l'utilisation de drogues injectables ou les pratiques de tatouage non sécuritaires (50 et données non publiées du Service correctionnel du Canada, 2015).

\section{Autochtones}

Les Autochtones semblent être touchés par l'infection par le VHC de manière disproportionnée : une enquête pilote auprès des Autochtones à Regina (Saskatchewan), réalisée de 2011 à 2012, a révélé que 42 \% des participants avaient été exposés au VHC dans leur vie (51-53). Quant aux Canadiens non Autochtones, l'utilisation de drogues injectables est le principal facteur de risque. D'autres déterminants sociaux pouvant rendre les Autochtones vulnérables à I'infection par le VHC incluent la pauvreté, un logement inadéquat et un manque d'accès à des services de soins de santé (51).

\section{Immigrants en provenance d'un pays à forte prévalence de VHC}

Certains pays ont une prévalence de VHC supérieure à la moyenne (plus de $3 \%$ ); les personnes nées dans ces régions ou y ayant habité pendant une période significative peuvent avoir un risque plus élevé d'infection par le virus de l'hépatite $C$, en particulier dans les régions où les mesures de prévention et de contrôle des infections dans les établissements de soins de santé ou de prestation de services personnels ne sont pas systématiquement mises en œuvre (54). Régions où l'hépatite C est plus courante : Asie centrale, Extrême-Orient, Asie du Sud, Australasie et Océanie, Europe de l'Est, Afrique subsaharienne et Afrique du Nord/Moyen-Orient $(4,55)$.

\section{Dépistage}

Le dépistage est une intervention viable en matière de santé publique lorsque les critères suivants sont satisfaits : la condition faisait l'objet du dépistage doit être un problème de santé important, l'évolution naturelle doit être bien comprise, il doit y avoir un stade précoce détectable, un test adapté doit être disponible pour les stades précoces, le traitement à un stade précoce doit être plus bénéfique qu'à un stade plus avancé, la disponibilité d'un test diagnostique doit être acceptée, des intervalles de tests répétés doivent être déterminés, des prestations des services de santé doivent être disponibles, les avantages doivent être supérieurs aux inconvénients, un traitement efficace doit être disponible et accessible, et enfin, le coût doit être compensé par les avantages (56).
Le dépistage de l'hépatite $\mathrm{C}$ chronique doit offrir des avantages pour la santé personnelle et la santé publique en interrompant la transmission virale (avantage public) et en réduisant la mortalité et la morbidité (avantage personnel). L'atteinte d'une réponse virologique soutenue est associée à un risque réduit d'événements hépatiques et à une vie plus saine pour les patients (57). De plus, un diagnostic précoce permet de formuler des recommandations visant à réduire la consommation d'alcool, ce qui peut ralentir la progression de la maladie du foie (58).

L'avantage pour la santé publique qui consiste à déterminer les cas non diagnostiqués par l'entremise d'un dépistage fondé sur les risques sert à prévenir l'acquisition et la transmission du VHC chez les nouveaux cas incidents. Des arguments sont avancés pour le dépistage de cohortes de naissance visant à déterminer les personnes souffrant d'hépatite $C$ chronique qui autrement ne recevraient pas de diagnostic. L'évaluation du GECSSP permettra de mieux comprendre l'applicabilité du dépistage de cohortes de naissance au Canada. On encourage les professionnels de la santé à évaluer le besoin en matière de dépistage du VHC dans le cadre de soins systématiques.

\section{Dépistage de l'hépatite $C$ fondé sur les risques}

Les recommandations actuelles en matière de dépistage de I'hépatite $\mathrm{C}$ au Canada sont fondées sur l'évaluation des facteurs de risque (59). Les risques associés à l'acquisition du VHC comprennent les activités impliquant tout risque d'exposition à du sang ou à des produits sanguins contaminés, notamment :

- Utilisation de drogues injectables ou partage de matériel d'utilisation de drogues contaminé, même une seule fois

- Réception de services de soins de santé ou personnels pour lesquels les pratiques de prévention et contrôle des infections ne sont pas bien appliquées

- Réception d'une transfusion sanguine, de produits sanguins ou d'une greffe d'organe avant 1992, au Canada

- Naissance ou résidence dans une région où l'hépatite $C$ est plus courante (prévalence supérieure à $3 \%$ ), notamment : Asie centrale, Extrême-Orient, Asie du Sud, Australasie et Océanie, Europe de l'Est, Afrique subsaharienne et Afrique du Nord/Moyen-Orient

- $\quad$ Autres risques :

- Partage d'articles de soins personnels avec une personne séropositive pour le virus de l'hépatite C

- Participation à une activité sexuelle à risque

○ Être né d'une mère séropositive pour le VHC

\section{Conclusion}

L'hépatite $C$ est une maladie complexe, car elle est infectieuse, peut demeurer asymptomatique pendant des décennies, peut se présenter sous la forme d'une maladie chronique et est associée à d'autres comorbidités chroniques. La présence de comorbidités peut avoir des répercussions sur le diagnostic, la prise en 
charge et la réussite du traitement de l'hépatite $C$ chronique. Grâce à la disponibilité de nouveaux traitements efficaces pour I'hépatite $C$, le dépistage de l'hépatite $C$ est important pour la détection précoce et pour améliorer la santé de la population et les résultats des patients. II faut connaître les facteurs de risque $d^{\prime}$ acquisition du VHC et identifier les personnes à risque afin de réduire la morbidité et la mortalité associées à l'hépatite $C$ et empêcher la retransmission.

\section{Conflit d'intérêts}

Aucun.

\section{Références}

1. Micallef JM, Kaldor JM, Dore GJ. Spontaneous viral clearance following acute hepatitis $C$ infection: a systematic review of longitudinal studies. J Viral Hepat. 2006;13(1):3441.

2. Chen $\mathrm{SL}$, Morgan TR. The natural history of hepatitis $\mathrm{C}$ virus (HCV) infection. Int J Med Sci. 2006;3(2):47-52.

3. Smith DB, Bukh J, Kuiken C, Muerhoff AS, Rice CM, Stapleton JT, et al. Expanded classification of hepatitis C virus into 7 genotypes and 67 subtypes: updated criteria and genotype assignment web resource. Hepatology. 2014;59(1):318-27.

4. Messina JP, Humphreys I, Flaxman A, Brown A, Cooke GS, Pybus OG, et al. Global distribution and prevalence of hepatitis C virus genotypes. Hepatology. 2015;61(1):77-87.

5. Statistics Canada. Table 102-0521. Deaths, by cause, Chapter I: Certain infectious and parasitic diseases (A00 to B99), age group and sex, Canada. Ottawa (ON): Statistics Canada; 2014.(Disponible en français : http://www5.statcan. gc.ca/cansim/a26?id=1020521\&pattern=death\&p2=1\&tabMode=dataTable\&p1 =1\&retrLang=fra\&srchLan=$1 \&$ lang=fra)

6. Mahajan R, Xing J, Liu SJ, Ly KN, Moorman AC, Rupp L, et al. Mortality among persons in care with hepatitis $C$ virus infection: the Chronic Hepatitis Cohort Study (CHeCS), 2006-2010. Clin Infect Dis. 2014;58(8):1055-61.

7. Webster G, Wu J, Williams B, Ivis F, de Sa E, Hall N. Canadian organ replacement register annual report: treatment of end-stage organ failure in Canada, 2003 to 2012. Ottawa (ON): Canadian Institute for Health Information; 2015. (Disponible en français : https://secure. cihi.ca/free_products/2014_CORR_Annual_Report_FR.pdf).

8. Kwong JC, Crowcroft NS, Campitelli MA, Ratnasingham S, Daneman N, Deeks SL, et al. Ontario Burden of Infectious Disease Study (ONBOIDS): an OAHPP/ICES report. Toronto (ON): ICES; 2010. (Disponible en français : https://www. publichealthontario.ca/fr/eRepository/ONBoID_ICES_ Report_ma18.pdf).

9. Smith BD, Morgan RL, Beckett GA, Falck-Ytter Y, Holtzman $D$, Teo CG, et al. Recommendations for the identification of chronic hepatitis $C$ virus infection among persons born during 1945-1965. MMWR Recomm Rep. 2012;61:1-32.
10. Moyer VA; U.S. Preventive Services Task Force. Screening for hepatitis $C$ virus infection in adults. Ann Intern Med. 2013; 159(5):349-57.

11. Schanzer DL, Paquette D, Lix LM. Historical trends and projected hospital admissions for chronic hepatitis $C$ infection in Canada: a birth cohort analysis. CMAJ Open. 2014;2(3):E139-44.

12. Trubnikov M, Yan $P$, Archibald C. Estimated prevalence of hepatitis C virus (HCV) infection in Canada, 2011. Can Commun Dis Rep. 2014:40;19. (Disponible en français : http://www.phac-aspc.gc.ca/publicat/ccdr-rmtc/14vol40/drrm40-19/surveillance-b-fra.php ).

13. Manns MP, McHutchison JG, Gordon SC, Rustgi VK, Shiffman M, Reindollar R, et al. Peginterferon alfa-2b plus ribavirin compared with interferon alfa-2b plus ribavirin for initial treatment of chronic hepatitis C: a randomised trial. Lancet. 2001;358(9286):958-65.

14. Kohli A, Shaffer A, Sherman A, Kottilil S. Treatment of hepatitis C: a systematic review. JAMA. 2014;312(6):631-40.

15. Health Canada. Drug and health products. HARVONI. Summary basis of decision (SBD). Ottawa (ON): Health Canada. (Disponible en français : http://www.hc-sc.gc.ca/ dhp-mps/prodpharma/sbd-smd/drug-med/sbd_smd_2015_ harvoni_173180-fra.php).

16. Kowdley KV, Gordon SC, Reddy KR, Rossaro L, Bernstein $D E$, Lawitz $E$, et al. Ledipasvir and sofosbuvir for 8 or 12 weeks for chronic HCV without cirrhosis. N Engl J Med. 2014;370(20):1879-88.

17. Poordad F, Hezode C, Trinh R, Kowdley KV, Zeuzem S, Agarwal K, et al. ABT-450/r-ombitasvir and dasabuvir with ribavirin for hepatitis $\mathrm{C}$ with cirrhosis. N Engl J Med. 2014;370(21):1973-82.

18. Centre for Communicable Diseases and Infection Control. Report on Hepatitis B and C in Canada: 2012. Ottawa (ON): Public Health Agency of Canada; 2012. (Disponible en français : http://canadiensensante.gc.ca/publications/ diseases-conditions-maladies-affections/hepatitis-b-c-2012hepatite-b-c/index-fra.php)

19. Trubnikov M, Yan P, Njihia J, Archibald C. Identifying and describing a cohort effect in the national database of reported cases of hepatitis $C$ virus infection in Canada (1991-2010): an age-period-cohort analysis. CMAJ Open. 2014;2(4):E281-7.

20. Remis RS. Modelling the incidence and prevalence of hepatitis $C$ infection and its sequelae in Canada, 2007. Final report. Ottawa (ON): Public Health Agency of Canada; 2007. (Disponible en français : http://www.phac-aspc.gc.ca/sti-itssurv-epi/model/pdf/model07-fra.pdf).

21. Rotermann M, Langlois K, Andonov A, Trubnikov M. Seroprevalence of hepatitis $B$ and $C$ virus infections: results from the 2007 to 2009 and 2009 to 2011 Canadian Health Measures Survey. Health Rep. 2013;24(11):1-13. 
22. Public Health Agency of Canada. Epidemiology of acute hepatitis $C$ infection in Canada: results from the Enhanced Hepatitis Strain Surveillance System (EHSSS). Ottawa (ON): Public Health Agency of Canada; 2009. (Disponible en français: http://publications.gc.ca/site/eng/349885/ publication.html).

23. Public Health Agency of Canada. Summary of key findings from I-Track Phase 3 (2010-2012). Ottawa (ON): The Agency; 2014. (Disponible en français: http://www.phac-aspc.gc.ca/ aids-sida/publication/reports/i-track-phase-3/assets/pdf/itrack-phase-3-fra.pdf).

24. Paintsil E, Binka M, Patel A, Lindenbach BD, Heimer R. Hepatitis $C$ virus maintains infectivity for weeks after drying on inanimate surfaces at room temperature: Implications for risks of transmission. J Infect Dis 2014;209(8):1205-11.

25. Page K, Morris MD, Hahn JA, Maher L, Prins M. Injection drug use and hepatitis $C$ virus in young adult injectors: using evidence to inform comprehensive prevention. Clin Infect Dis. 2013;57(S2):S32-8.

26. Su Y, Norris JL, Zang C, Peng Z, Wang N. Incidence of hepatitis $C$ virus infection in patients on hemodialysis: a systematic review and meta-analysis. Hemodial Int. 2013;17(4):532-41.

27. Westermann C, Peters C, Lisiak B, Lamberti M, Nienhaus A. The prevalence of hepatitis $C$ among healthcare workers: $a$ systematic review and meta-analysis. Occup Environ Med. 2015;72(12):880-8.

28. Jafari S, Copes R, Baharlou S, Etminan M, Buxton J. Tattooing and the risk of transmission of hepatitis $\mathrm{C}$ : a systematic review and meta-analysis. Int J Infect Dis. 2010;14(11):e928-40.

29. Bianco A, Bova F, Nobile CG, Pileggi C, Pavia M, Collaborative Working Group. Healthcare workers and prevention of hepatitis $C$ virus transmission: exploring knowledge, attitudes and evidence-based practices in hemodialysis units in Italy. BMC Infect Dis. 2013;13(76).

30. Yang J, Hall K, Nuriddin A, Woolard D. Risk for hepatitis B and $C$ virus transmission in nail salons and barbershops and state regulatory requirements to prevent such transmission in the United States. J Public Health Manag Pract. 2014;20(6):E20-30.

31. CBC News. Hepatitis $C$ outbreak identified at Kitchener colonoscopy clinic. 2015; Kitchener-Waterloo. http:// www.cbc.ca/news/canada/kitchener-waterloo/hepatitisc-outbreak-identified-at-kitchener-colonoscopyclinic-1.294360332.

32. Region of Waterloo. Public Health and Emergency Services. Hepatitis C - Tri-City Colonoscopy Clinic Investigation. 2015 Feb 3. http://chd.region.waterloo.on.ca/en/ healthyLivingHealthProtection/resources/PHE_IDS_15_02_ HEPC.pdf.

33. Toronto Metro. Experts warn against use of multi-dose vials after hepatitis C outbreak at Toronto clinics. News/Canada. http://www.metronews.ca/news/canada/2014/09/30/ experts-warn-against-use-of-multi-dose-vials-after-hepatitisc-outbreak-at-toronto-clinics.html.
34. Boyle T. Hepatitis C outbreaks at three Toronto colonoscopy clinics kept secret. The Toronto Star. 2014 Sep 27; Life/Health \& Wellness. http://www.thestar.com/life/health_ wellness/2014/09/27/hepatitis_c_outbreaks_at_three_ toronto_colonoscopy_clinics_kept_secret.html.

35. Ruby M. Test confirms patient infected with hepatitis C during May 29, 2013, procedure at BGH. Brantford Expositor. 2014 Jan 29; News Brantford-Brant http://www. brantfordexpositor.ca/2014/01/29/test-confirms-patientinfected-with-hepatitis-c-during-may-29-2013-procedure-atbgh.

36. Canadian Blood Services: surveillance report, 2014. Ottawa (ON): Canadian Blood Services; 2014. https://www.blood.ca/ sites/default/files/blood/blood-safety/External-SurveillanceReport-2014.pdf.

37. Benova L, Mohamoud YA, Calvert C, Abu-Raddad LJ. Vertical transmission of hepatitis $C$ virus: systematic review and meta-analysis. Clin Infect Dis. 2014;59(6):765-73.

38. Robinson JL; Canadian Pediatric Society. Vertical transmission of hepatitis $C$ virus: current knowledge and issues. Ottawa (ON): Canadian Pediatric Society; 2014. (Disponible en français : http://www.cps.ca/fr/documents/ position/transmission-verticale-du-virus-de-hepatite-C).

39. Terrault NA, Dodge JL, Murphy EL, Tavis JE, Kiss A, Levin $T R$, et al. Sexual transmission of hepatitis $C$ virus among monogamous heterosexual couples: the HCV partners study. Hepatology. 2013;57(3):881-9.

40. Witt MD, Seaberg EC, Darilay A, Young S, Badri S, Rinaldo $C R$, et al. Incident hepatitis $C$ virus infection in men who have sex with men: a prospective cohort analysis, 1984-2011. Clin Infect Dis. 2013;57(1):77-84.

41. Wong J, Moore D, Kanters S, Buxton J, Robert W, Gustafson $\mathrm{R}$, et al. Seroprevalence of hepatitis $C$ and correlates of seropositivity among men who have sex with men in Vancouver, Canada: a cross-sectional survey. Sex Transm Infect 2015;91(6):430-3.

42. Foster AL, Gaisa M, Hijdra RM, Fierer DS, Jacobson K, Turner S, et al. Rectal shedding of HCV in HCV/HIV co-infected men.; San Francisco (CA): AASLD Liver Meeting 2015; 2015 Nov 13-17. Abstract 89.

43. Burchell AN, Gardner SL, Mazzulli T, Manno M, Raboud J, Allen VG, et al. Hepatitis $C$ virus seroconversion among HIV-positive men who have sex with men with no history of injection drug use: Results from a clinical HIV cohort. Can J Infect Dis Med Microbiol. 2015;26(1):17-22.

44. Yaphe S, Bozinoff N, Kyle R, Shivkumar S, Pai NP, Klein M. Incidence of acute hepatitis $C$ virus infection among men who have sex with men with and without HIV infection: a systematic review. Sex Transm Infect. 2012;88(7):558-64.

45. Apers L, Vanden Berghe W, De Wit S, Kabeya K, Callens S, Buyze J, et al. Risk factors for HCV acquisition among HIV-positive MSM in Belgium. J Acquir Immune Defic Syndr. 2015;68(5):585-93. 
46. Breskin A, Drobnik A, Pathela P, Chan C, Braunstein S, Bornschlegel $\mathrm{K}$. Factors associated with hepatitis $C$ infection among HIV-infected men who have sex with men with no reported injection drug use in New York City, 2000-2010. Sex Transm Dis. 2015;42(7):382-6.

47. Reinhart J. Sexual transmission of hepatitis C: are HIVpositive gay and bisexual men at risk? Toronto (ON): CATIE; 2011. (Disponible en français : http://www.catie.ca/fr/ pdm/printemps-2011/transmission-sexuelle-hepatite-c-leshommes-seropositifs-gais-bisexuels-sont-ils-).

48. Zakaria D, Thompson JM, Jarvis A, Smith J. Testing and treatment for human immunodeficiency virus and hepatitis $C$ virus infections among Canadian federal inmates. Ottawa (ON): Correctional Service of Canada; 2010. (Disponible en français : http://www.csc-scc.gc.ca/005/008/092/0050080223-01-fra.pdf).

49. Correctional Services Canada. Bloodborne and sexually transmitted infections in Canadian federal penitentiaries: program overview. In: Ontario HIV/AIDS Trials Network Conference; 2014 Nov 30. Kingston, ON.

50. Wenger PJ, Rottnek F, Parker T, Crippin JS. Assessment of Hepatitis C Risk Factors and Infection Prevalence in a Jail Population. Am J Public Health. 2014 September; 104(9): 1722-1727.

51. Public Health Agency of Canada. Summary of key findings from the A-Track pilot survey (2011-2012). Ottawa (ON): 2013. (Disponible en français : http://publications.gc.ca/ collections/collection_2014/aspc-phac/HP40-118-2014-fra. pdf).

52. Frescura AM, Fang L, Trubnikov M, Klar S, Jayaraman G. Centre for Communicable Diseases and Infection Control. Hepatitis C in Canada: 2005-2010 surveillance report. Ottawa (ON): Public Health Agency of Canada; 2012. (Disponible en français : http://www.healthycanadians. gc.ca/publications/diseases-conditions-maladies-affections/ hepatitis-b-c-2012-hepatite-b-c/index-fra.php).
53. Spittal PM, Pearce ME, Chavoshi N, Christian WM, Moniruzzaman A, Teegee M, et al. The Cedar Project: high incidence of HCV infections in a longitudinal study of young Aboriginal people who use drugs in two Canadian cities. BMC Public Health. 2012;12:632.

54. Greenway C Ma AT, Kloda LA, Klein MB, Cnossen S. The seroprevalence of hepatitis $C$ in immigrants and refugees from intermediate and high endemic countries: a systematic review and meta-analysis. Gastroenterology. 2015;148(4;S1):S998-9.

55. Smith B, Falck-Ytter Y; Guidelines Development Group. Guidelines for the screening, care and treatment of persons with hepatitis $\mathrm{C}$ infection. Geneva $(\mathrm{CH})$ : World Health Organization; 2014 Apr. http://apps.who.int/iris/ bitstream/10665/111747/1/9789241548755_eng.pdf.

56. Wilson JM, Jungner G. Principles and practice of screening for disease. Geneva (CH): World Health Organization; 1968.

57. Innes HA, McDonald SA, Dillon JF, Allen S, Hayes PC, Goldberg D, et al. Toward a more complete understanding of the association between a hepatitis $C$ sustained viral response and cause-specific outcomes. Hepatology. 2015;62(2):355-64

58. Zule WA, Costenbader EC, Coomes CM, Wechsberg WM. Effects of a hepatitis $C$ virus educational intervention or a motivational intervention on alcohol use, injection drug use, and sexual risk behaviours among injection drug users. Am J Public Health. 2009;99(S1):S180-6.

59. Public Health Agency of Canada. Primary care management of chronic hepatitis C: professional desk reference 2009. Mississauga (ON): College of Family Physicians of Canada; 2009. Joint publication of the Public Health Agency of Canada. http://www.catie.ca/sites/default/files/Primary-CareManagement-of-Chronic-Hepatitis-C-Professional-DeskReference.pdf 